\title{
ESTUDO COMPARATIVO DA FORMAÇÃO DO CIRCUITO ARTERIOSO DO CÉREBRO DE COELHOS, GATOS E EQUINOS
}

\author{
Marcelo Salvador Gomes ${ }^{1}$ \\ Carlos Augusto dos Santos-Sousa ${ }^{2}$ \\ Paulo Souza Junior \\ Marcelo Abidu-Figueiredo
}

GOMES, M. S.; SANTOS-SOUSA, C. A. dos; SOUZA JUNIOR, P.; ABIDU-FIGUEIREDO, M. Estudo comparativo da formação do circuito arterioso do cérebro de coelhos, gatos e equinos. Arq. Ciênc. Vet. Zool. UNIPAR, Umuarama, v. 22, n. 1, p. 1-6, jan./mar. 2019.

RESUMO: O estudo das artérias responsáveis pela irrigação do encéfalo nos animais, assim como seus aspectos filogenéticos são motivos de interesse na pesquisa biomédica devido à grande variabilidade dos padrões descritos na literatura. O objetivo desta investigação foi caracterizar comparativamente a formação do circuito arterioso do cérebro em coelhos, gatos e equinos adultos mestiços de ambos os sexos. Os cadáveres dos animais foram fixados através de uma solução de formaldeído a $10 \%$ por meio de canulação na artéria carótida comum. Em seguida, foram feitas repleções vasculares com solução aquosa de Petrolátex S65 corado com pigmento Suvinil nas cores vermelho, azul e verde. Para esta pesquisa, foram utilizadas 44 cabeças de coelhos da Nova Zelândia, 50 de gatos SRD e 30 de equinos mestiços. Procedeu-se a craniotomia, remoção dos encéfalos e dissecção para a observação da formação do circuito arterioso. Nos coelhos, o circuito arterioso do cérebro estava fechado rostralmente em 10 machos e 12 fêmeas e fechado caudalmente em todos os animais dissecados. Em gatos o circuito arterioso do cérebro apresentou-se aberto rostralmente em nove machos e oito fêmeas e caudalmente esteve fechado em todos os animais dissecados. Nos equinos o circuito arterioso do cérebro apresentou-se fechado rostral e caudalmente em $100 \%$ dos animais dissecados. Em todos os espécimes utilizados no estudo, as artérias da base do encéfalo estiveram na dependência dos sistemas carótico e vértebrobasilar.

PALAVRAS-CHAVE: Artérias. Dissecção. Vascularização do encéfalo.

\section{COMPARATIVE STUDY ON THE FORMATION OF THE CEREBRAL ARTERIAL CIRCUIT OF RABBITS, CATS AND HORSES}

\begin{abstract}
The blood supply of the brain and its phylogenetic aspects are of great interest in biomedical research, since the patterns presented in literature are subject to numerous variations. The aim of this study was to comparatively describe the formation of the arterial circuit of the brain in adult rabbits, cats and crossbreed horses of both sexes. The cadavers were fixated in a $10 \%$ formalin solution by cannulation of the common carotid artery. Afterwards, the arteries were injected with a latex solution dyed in red, blue and green. For this research, 44 heads of New Zealand rabbits, 50 heads of non-defined breed cats, and 30 heads of crossbred horses were used. The specimens were submitted to craniotomy and the arterial supply of the brain was dissected. In rabbits, the arterial brain circuit was rostrally closed in 10 males and 12 females, and caudally closed in all dissected animals. In cats, the brain circuit was rostrally open in 09 males and 08 females, and caudally closed in $100 \%$ of the sample. The brain circuit was rostrally and caudally closed in all horses. The brain base arteries were dependent on the carotid and vertebral-basilar systems in all specimens used in this study.
\end{abstract}

KEY WORDS: Arteries. Brain vascularization. Dissection.

\section{ESTUDIO COMPARATIVO DE LA FORMACIÓN DEL CIRCUITO ARTERIOSO DEL CEREBRO DE CONEJOS, GATOS Y EQUINOS}

RESUMEN: El estudio de las arterias responsables por la irrigación del encéfalo en animales, así como sus aspectos filogenéticos, son motivos de interés en la investigación biomédica debido a la gran variabilidad de los patrones descritos en la literatura. El objetivo de esta investigación ha sido caracterizar comparativamente la formación del circuito arterial del cerebro en conejos, gatos y equinos adultos mestizos de ambos sexos. Los cadáveres de los animales se fijaron mediante una solución de formaldehído al $10 \%$ por medio de la canulación en la arteria carótida común. A continuación, se realizaron replementos vasculares con solución acuosa de Petrolátex S65 colorado con pigmento Suvinil en los colores rojo, azul y verde. Para esta investigación, se utilizaron 44 cabezas de conejos de Nueva Zelanda, 50 de gatos SRD y 30 de equinos mestizos. Se procedió a la craneotomía, remoción de los encéfalos y disección para la observación de la formación del circuito arterioso. En los conejos, el circuito arterioso del cerebro estaba cerrado rostralmente en 10 machos y 12 hembras y cerrado caudalmente en todos los animales disecados. En gatos el circuito arterioso del cerebro se presentó abierto rostralmente en nueve machos y ocho hembras y caudalmente estuvo cerrado en todos los animales disecados. En los equinos el circuito arterioso del cerebro

DOI: 10.25110 /arqvet.v22i1.2019.7159

'Docente do Departamento de Anatomia Animal e Humana da Universidade Federal Rural do Rio de Janeiro (UFRRJ). salvador-marcelo@hotmail.com

${ }^{2}$ Docente da Área de Anatomia Animal da Universidade Federal do Acre (UFAC).

${ }^{3}$ Docente da Área de Anatomia Animal da Universidade Federal do Pampa (UNIPAMPA). 
se presentó cerrado rostral y caudalmente en el 100\% de los animales disecados. En todos los especímenes utilizados en el estudio, las arterias de la base del encéfalo estuvieron en la dependencia de los sistemas caróxicos y vértebrobasilar.

PALABRAS CLAVE: Arterias. Disección. Vascularización del encéfalo.

\section{Introdução}

As doenças que acometem o sistema nervoso central dos animais domésticos e silvestres representam grande parcela das afecções observadas na prática clinico-cirúrgica desses animais e podem ser decorrentes de deficiência de irrigação e consequente lesão isquêmica (MENESES, 2011).

A abordagem cirúrgica do encéfalo requer um conhecimento anatômico preciso, não só da habitual morfologia, mas também das variações das diversas estruturas vasculares que se relacionam com o acesso cirúrgico (PARK; LEE; HONG, 2016).

O estudo das artérias responsáveis pela irrigação do encéfalo nos animais domésticos e silvestres e seus aspectos filogenéticos continuam sendo motivos de interesse na pesquisa biomédica. Isso se dá devido à grande variabilidade dos arranjos apresentados pelas artérias formadoras do "círculo de Willis" (BARONE; BORTOLAMI, 2004; DE VRIESE, 1905; PRADA, 2014; TESTUT, 1911), embora esses diferentes aspectos morfológicos não representem necessariamente parâmetros evolutivos (DE VRIESE, 1905). Dessa maneira, acredita-se que o comportamento das artérias encefálicas, comparativamente entre as diferentes espécies, exibe um modelo básico ao qual são acrescentadas modificações relativas aos diferentes grupos de animais (DE VRIESE, 1905; TESTUT, 1911).

Tais artérias, assim como o encéfalo, estão sob processo contínuo de modificações, o que caracteriza a existência de um paralelismo entre a evolução do sistema nervoso central e as constantes modificações na configuração dos vasos responsáveis por sua vascularização. Portanto, suas relações filogenéticas e ontogênicas de seus modelos arquitetônicos e anastomóticos podem auxiliar o entendimento dessas modificações (LIMA et al., 2006).

Testut (1911) afirma que a única artéria que conduz sangue à massa encefálica primitivamente é a artéria carótida interna, sendo que a artéria vertebral não existe. No estágio médio embrionário, as duas artérias cerebrais anteriores, até então independentes, unem-se na linha média, ora por meio de uma rede intermediária, ora por meio de um simples ramo transversal, que representa a artéria comunicante anterior. Segundo o mesmo autor, os dois ramos da artéria carótida interna, logo atrás da origem da futura artéria cerebral posterior, fundem-se em um tronco único mediano, o tronco basilar. Já, no estágio final, a parte anterior do ramo caudal da artéria carótida interna sofre uma atrofia; a artéria vertebral aumenta de volume e supre assim a artéria basilar, onde o sangue passa então a circular de baixo para cima, acontecendo o mesmo com seus ramos de bifurcação anterior (artérias cerebrais anteriores) que parecem continuá-la.

O presente artigo teve como objetivo caracterizar comparativamente a formação do circuito arterioso em equinos mestiços, gatos sem raça definida (SRD) e coelhos Nova Zelândia adultos de ambos os sexos.

\section{Material e Métodos}

Os estudos de dissecção anatômica foram realizados em 124 cabeças de cadáveres adultos, sendo 44 coelhos Nova Zelândia (21 machos e 23 fêmeas), 50 gatos SRD (25 machos e 25 fêmeas) e 30 equinos mestiços ( 15 machos e 15 fêmeas). Os cadáveres de coelhos eram provenientes do descarte de experimentos prévios e os de felinos e equinos doados após morte natural em Hospitais e Clínicas Veterinárias. As cabeças foram previamente identificadas e, por meio de uma cânula plástica introduzida na artéria carótida comum, o sistema arterial foi "lavado" com solução fisiológica de $\mathrm{NaCl}$ a $0,9 \%$, sendo realizada a seguir, a fixação com solução de formaldeído a 10\%, conforme técnica anatômica padrão. Em seguida, injetou-se pela cânula solução aquosa (diluição 1:1) de Petrolátex S-65 (Refinaria Duque de Caxias (REDUC) da Petrobrás, Duque de Caxias-RJ) misturado ao corante (Suvinil xadrez $\left.{ }^{\circledR}\right)$.

Posteriormente, as calotas cranianas foram excisadas e incisões foram feitas nas meninges, mergulhando-se em seguida todo o material em solução de formol a 30\%, por cinco dias, para complementação da fixação dos encéfalos e coagulação do látex. Para abertura dos crânios, remoção dos encéfalos e dissecação dos vasos da base, foram utilizados instrumentais cirúrgicos de rotina e fórceps odontológico.

O registro dos arranjos vasculares observados foram classificados de acordo com o proposto por De Vriese (1905) para vertebrados, em três tipos: tipo I, no qual a irrigação cerebral é quase exclusivamente feita através de ambas as artérias carótidas internas; tipo II, onde o encéfalo é parcialmente irrigado pela carótida interna e pelo sistema vertebrobasilar, com igual distribuição entre os dois sistemas ou a predominância de um deles; e tipo III, em que o encéfalo é irrigado quase exclusivamente pelo sistema vertebrobasilar.

Em particular, o tipo II apresenta dois subtipos: no sub-tipo 2 alfa, os ramos terminais caudais (artéria comunicante caudal), que se originam nas artérias carótida interna, não mais se implantam na artéria basilar, pois terminam nas artérias cerebrais caudais e sofrem anastomose com os ramos da artéria basilar, resultante da união da artérias vertebrais. No sub-tipo 2 beta a artéria basilar se mostra mais importante, sendo as artérias cerebrais caudais, a continuação direta de seus ramos os quais sofrem anastomoses com ramos terminais caudais (artéria comunicante caudal) das artérias carótidas interna, menos desenvolvidas que no sub-tipo 2 alfa.

Assim, os vasos da base do encéfalo das três espécies foram dissecados, classificados conforme proposto por De Vriese (1905), fotografados para documentação e denominados de acordo com a International Committee on Veterinary Gross Anatomical Nomenclature (2017).

\section{Resultados}

\section{Coelhos}

O circuito arterioso do cérebro do coelho envolveu rostralmente a base dos nervos ópticos, o túber cinéreo e, caudalmente, o corpo mamilar e a fossa interpeduncular. Es- 
tendeu-se desde a fissura longitudinal do cérebro na altura da base do trígono olfatório até o sulco que separa a ponte dos pedúnculos cerebrais.

Os vasos que o delimitavam foram: rostralmente, ramo rostral da artéria carótida interna, artérias cerebrais rostrais e artéria comunicante rostral; e caudalmente, pelos ramos caudais da artéria carótida interna (artérias comunicantes caudais) e os ramos terminais da artéria basilar (Figura 1).

O circuito arterioso do cérebro apresentou-se fechado rostralmente pela presença da artéria comunicante rostral em 10 (47,62\%) machos e 12 (52,17\%) fêmeas. Esse circuito encontrou-se fechado caudalmente em todos os animais dissecados. Em nenhuma das amostras o circuito arterioso assemelhou-se a uma figura geométrica.

$\mathrm{O}$ arranjo geral das artérias da base do encéfalo nos coelhos pode ser caracterizado como tipo II (De Vriesse 1905), pois foi formado por meio dos sistemas carótico e vértebro-basilar. Outrossim, apresentaram tendência ao subtipo II beta.

Figura 1: Fotomacrografia da vista ventral do circuito arterioso do coelho: acr - artéria cerebral rostral, acm - artéria cerebral média, rrci - ramo rostral da carótida interna, rcci - ramo caudal da carótida, qo - quiasma óptico, tc - túber cinério, * e pc - pedúnculos cerebrais direito e esquerdo, po - ponte, ab - artéria basilar.

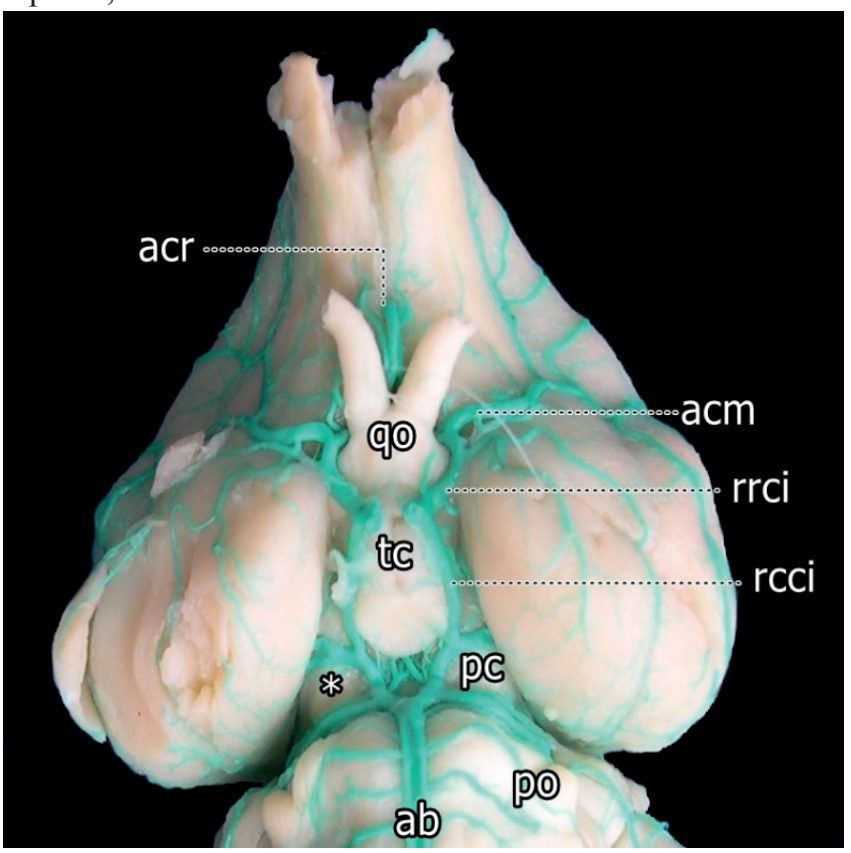

\section{Equinos}

O circuito arterial da base do encéfalo equino foi formado pelas artérias: comunicante caudal direita e esquerda, carótida interna em ambos os antímeros e cerebral rostral direita e esquerda (Figura 2). Caudalmente, apresentou-se fechado em $100 \%$ dos animais e configurado pelas artérias comunicante caudal direita e esquerda. Rostralmente, apresentou se fechado em $100 \%$ dos animais e arquitetado pelas artérias cerebral rostral direita e esquerda. Ainda rostralmente, a artéria cerebral rostral fusionou-se, formando a artéria comum do corpo caloso, onde o circuito arterial se fechou. Em nenhuma das amostras o circuito arterioso se assemelhou a uma figura geométrica.
O arranjo geral das artérias da base do encéfalo nos equinos está na dependência dos sistemas carótico e vértebro-basilar, o que caracteriza o tipo II da classificação de De Vriesse (1905). Ademais, apresentaram tendência ao subtipo II beta.

Figura 2: Fotomacrografia da vista ventral do circuito arterioso de equino: accc - artéria comum do corpo caloso, acm - artéria cerebral média, acc - artéria comunicante cauldal, rcci - ramo caudal da carótida interna, qo - quiasma óptico, po - ponte, acb - artéria caróticobasilar, ab - artéria basilar, acrc - artéria cerebelar caudal.

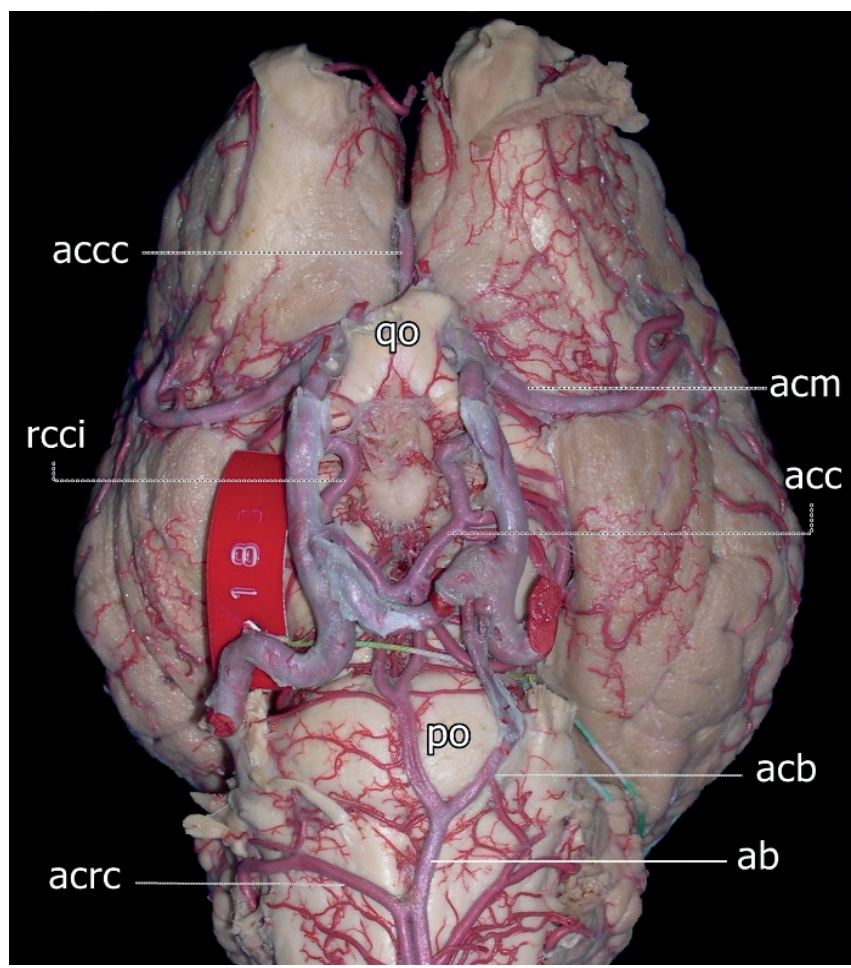

Gatos

Os vasos que caracterizam o circuito arterioso encefálico circundaram o corpo mamilar, a fossa interpeduncular, o túber cinéreo, a hipófise e o quiasma óptico.

$\mathrm{O}$ circuito arterial do cérebro de gatos estende-se desde a ponte até rostralmente ao quiasma óptico, sendo representado pela artéria carótida do encéfalo, direita e esquerda, por meio de seus correspondentes ramos rostrais e caudais e, ainda pelos ramos terminais, de ambos os antímeros e da artéria basilar (Figura 3)

O circuito arterioso do cérebro apresentou-se aberto rostralmente em nove machos e oito fêmeas, caudalmente esteve fechado em todos os animais dissecados. Em nenhuma das amostras o circuito arterioso se assemelhou a uma figura geométrica.

O arranjo geral das artérias da base do encéfalo nos gatos está na dependência dos sistemas carótico e vértebro-basilar, o que caracteriza o tipo II da classificação de De Vriesse (1905). Além de apresentarem tendência aos subtipos II alfa e beta. 
Figura 3: Fotomacrografia da vista ventral do circuito arterioso do gato: acr - artéria cerebral rostral, acm - artéria cerebral média, rrci - ramo rostral da carótida interna, rcci ramo caudal da carótida interna, to - tratos ópticos, tc - túber cinério, * e pc - pedúnculos cerebrais direito e esquerdo, rtab - ramos terminais da artéria basilar, po - ponte, ab - artéria bassilar.

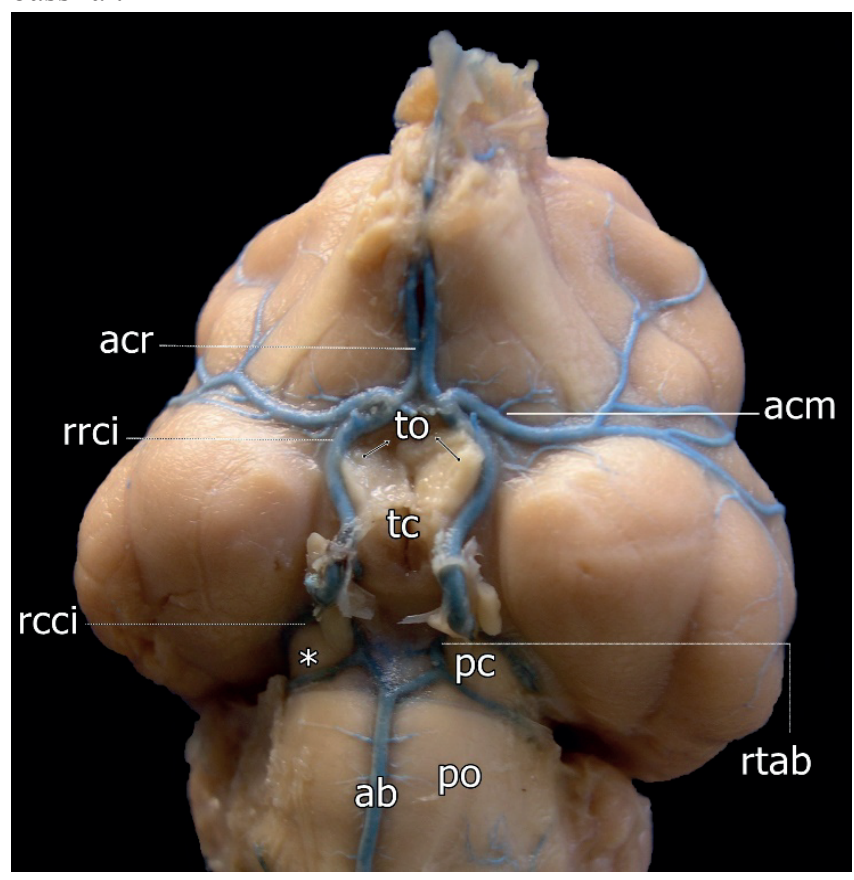

\section{Discussão}

De acordo com a classificação morfológica para o estudo das artérias da base do encéfalo, foi observado que os gatos do presente estudo apresentaram tendência ao subtipo 2 alfa ao subtipo 2 beta, e encontram-se entre os estágios médio e final de seu desenvolvimento filogenético. Já os equinos e coelhos apresentaram tendência ao subtipo 2 beta, e encontram-se entre os estágios médio e final de seu desenvolvimento filogenético.

O circuito arterioso do cérebro em animais domésticos e selvagens pode ser usado para criar modelos que simulem o homem, com o propósito de conduzir diferentes experimentos. Sendo assim, o estudo morfológico comparativo detalhado desses vasos torna-se essencial e relevante. Estudos do circuito arterioso da base do encéfalo de diferentes espécies demonstram muitas variações.

Nazer e Campos (2011), em avestruz (Struthio camelus) observaram que este se apresentou sempre aberto rostralmente e, em $80 \%$ dos casos aberto caudalmente. Almeida e Campos (2011), no jacaré (Caiman latirostris) verificaram que este foi dependente exclusivamente do sistema carótico, e se apresentou-se fechado rostralmente e caudalmente em todos os espécimes estudados, semelhante aos resultados obtidos por Azambuja, Goltz e Campos (2018), em estudo com o Ratão do Banhado (Myocastor coypus), embora o circuito nesses animais fosse suprido exclusivamente pelo sistema vértebro-basilar. Na presente investigação, o circuito arterioso no coelho mostrou-se fechado rostralmente pela presença da artéria comunicante rostral em 10 machos e 12 fêmeas, fechado caudalmente em $100 \%$ e na dependência exclusiva do sistema carótico e vértebro-basilar; em equinos o circuito apresentou-se fechado rostralmente e caudalmente em todos os espécimes analisados na dependência do sistema carótico e vértebro-basilar. Em gatos o circuito se mostrou aberto rostralmente em nove machos e oito fêmeas, fechado caudalmente em $100 \%$ e na dependência do sistema carótico e vértebro-basilar semelhante em parte aos resultados obtidos por Gomes et al. (2015), Lima et al. (2006), Moraes et al. (2014) e Portugal et al. (2014).

O circuito arterioso do suíno (Sus scrofa domesticus) é fechado rostralmente pela presença constante da artéria comunicante rostral em $100 \%$ dos casos e caudalmente pela presença dos ramos terminais direito e esquerdo da artéria basilar (FERREIRA; PRADA, 2005) semelhante ao observado no Javali (Sus scrofa scrofa) (OLIVEIRA; CAMPOS, 2005) e, diferentemente em parte dos observados na presente investigação.

No gato Gomes et al. (2015) e Lima et al. (2006) observaram que o circuito se mostrou fechado rostralmente pela presença da artéria comunicante rostral em $60 \%$ dos casos e caudalmente fechado pela presença dos ramos caudais das artérias carótidas do encéfalo e ramos terminais da artéria basilar em todas as amostras, semelhante ao observado na presente pesquisa.

Ocal et al. (1999), em camelos (Camelus dromedarius), concluíram que o circuito arterioso teve seu suprimento sanguíneo oriundo da artéria carótida, em cada antímero, da artéria basilar além da artéria comunicante caudal que se originou da rede admirável epidural rostral. Apesar das semelhanças a respeito da formação do circuito (a. carótida e a. basilar) nos coelhos, gatos e equinos desse presente estudo, a artéria comunicante caudal foi encontrada somente nos equinos.

Araújo e Campos (2005), em chinchilas (Chinchilla lanígera), relataram que a irrigação sanguínea do encéfalo é formada pelo sistema vértebro-basilar, porém em pequenas proporções, pela artéria carótida interna esquerda ou pelo sistema-vértebro basilar com contribuição da artéria carótida interna esquerda. Reckziegel; Lindemann e Campos (2001) verificaram que o circuito depende unicamente do sistema vértebro-basilar em capivaras (Hydrochoerus hydrochaeris), o que difere dos resultados encontrados no presente estudo, em que além da participação do sistema vértebro-basilar pôde-se observar a participação do sistema carótico.

No circuito arterioso do porco-espinho (Hystrix cristata), esquilo (Spermophilus citellus) e esquilo vermelho (Sciurus vulgaris), a artéria carótida interna não contribui para o suprimento sanguíneo do circuito arterioso, sendo esse feito pela artéria basilar (AYDIN et al., 2005; AYDIN et al., 2008; AYDIN et al., 2009). Em contrapartida, os presentes resultados encontraram que além da artéria basilar, há participação da artéria carótida interna. Lima et al. (2006) e Gomes et al. (2015), mostraram em gatos SRD que o circuito arterioso estende-se desde a ponte até o quiasma óptico (rostralmente), sendo representado pela artéria carótida interna direita e esquerda, por meio de seus correspondentes ramos rostrais e caudais e, ainda pelos ramos terminais em ambos os antímeros da artéria basilar em todas as preparações, corroborando com os resultados da presente investigação.

Em relação à forma do circuito arterioso em gatos, Lima et al. (2006) relataram que o arranjo dos ramos rostrais da carótida lembram uma figura elipsoide, disposta trans- 
versalmente em relação à base do encéfalo. Diferentemente, Gomes et al. (2012), também em gatos, observaram que o circuito arterioso não apresentou nenhuma configuração semelhante a uma forma geométrica. Na presente investigação o circuito arterioso nas três espécies estudadas não formaram nenhuma forma geométrica.

\section{Conclusões}

A comparação da dissecção dos encéfalos das três espécies permitiu inferir que os equinos têm o circuito arterioso invariavelmente fechado, enquanto nos felinos e coelhos variações podem ocorrer com maior frequência.

\section{Agradecimentos}

Agradecimento à Fundação de Amparo a Pesquisa do Estado do Rio de Janeiro (FAPERJ) e ao Conselho Nacional de Desenvolvimento Científico e Tecnológico - CNPq pelo financiamento.

\section{Referências}

ALMEIDA, L.; CAMPOS, R. A. Systematic study of the brain base arteries in broad-snouted caiman (Caiman latirostris). Journal of Morphological Science, São Paulo, v. 28, n. 1, p. 62-68, 2011.

ARAÚJO, A. C. P.; CAMPOS, R. A systematic study of the brain base arteries and their blood supply sources in the chinchilla (Chinchilla lanigera Molina, 1782). Brazilian Journal of Morphological Science, São Paulo, v. 22, n. 4, p. 221-232, 2005

AYDIN, A. et al. The morphology of circulus arteriosus cerebri in the porcupine (Hystrix cristata). Veterinárni Medicina, Praga, v. 50, n. 3, p. 131- 135, 2005.

AYDIN, A. et al. Morphological Investigations on the Circulus Arteriosus Cerebri in MoleRats (Spalax leucodon). Anatomia, Histologia, Embryologia, Berlin, v. 37, n. 3, p. 219-222, 2008.

AYDIN, A. et al. Morphology of the circulus arteriosus cerebri in the ground squirrel (Spermophilus citellus). Veterinárni Medicina, Praga, v. 54, n. 11, p. 537-542. 2009.

AZAMBUJA, R. C.; GOLTZ, L. V.; CAMPOS, R. Sistematização das artérias da base do encéfalo e suas fontes de suprimento sanguíneo em nutria (Myocastor coypus). Acta Scientiae Veterinaria, Porto Alegre, v. 46, p. 1-9, 2018.

BARONE, R.; BORTOLAMI, R. Anatomie comparée des Mammifères domestiques. Paris: Vigot, 2004. 1052p.

DE VRIESE, B. Sur la signification morphologique des artères cerebrales. Archives de Biologie, Pisa University Press, v. 21, p. 357-457, 1905

FERREIRA, C. G.; PRADA, I. L. S. O circuito arterial da base do encéfalo em suínos (Sus scrofa domesticus, Linnaeus,
1758), formação e comportamento. Brazilian Journal of Veterinary Research and Animal Science, São Paulo, v. 42, n. 1, p. 53-60, 2005.

GOMES, M. S. et al. Circulus arteriosus formation in cat brain. Eurasian Journal of Veterinary Science, Selcuk University Press, 28, n.. 4, p. 204-208, 2012.

GOMES, M. S. et al. Craniometria, morfometria do encéfalo e tipologia do circuito arterial da base do encéfalo em gatos. Revista Brasileria de Ciencia Veterinária, Niterói, v. 22, n. 1, p. 3-9, 2015.

INTERNATIONAL COMMITTEE ON VETERINARY GROSS ANATOMICAL NOMENCLATURE Nomina Anatomica Veterinária. 6. ed. Hannover: Columbia, Gent, Sapporo: Editorial Committee, 2017. 178p.

LIMA, E. M. M. et al. Estudo anatômico das artérias da base do encéfalo em gatos (Felis catus domesticus). Ars Veterinaria, Jaboticabal, v. 22, n. 1, p. 1-7. 2006.

MENESES, S. M. Neuroanatomia aplicada. Rio de Janeiro: Guanabara Koogan, 2011. 368p.

MORAES, S. O. S. et al. Morfometria do encéfalo e formação do circuito arterial em equinos mestiços. Ciência Animal Brasileira, Goiânia, v. 15, n. 3, p. 330-338, 2014.

NAZER, M. B.; CAMPOS, R. Systematization of the brain base arteries in ostrich (Struthio camelus). Journal of Morphoogical Science, São Paulo, v. 28, n. 4, p. 268-274, 2011.

OCAL, M. K. et al. A Quantitative study of the circulus arteriosus cerebri of the camel (Camelus dromedarius). Anatomia, Histologia, Embryologia, Berlin, v. 28, n. 5-6, p. 271-272, 1999 .

OLIVEIRA, J. C. D.; CAMPOS, R. A systematic study of brain base arteries in the wild boar (Sus scrofa scrofa). Anatomia, Histologia, Embryologia, Berlin, v. 34, n. 4, p. 232-239, 2005.

PARK, H. H.; LEE, K. S.; HONG, C. K. Vertebral artery transposition via na extreme-lateral approach for anterior foramen Magnum meningioma or craniocervical junction tumors. World Neurosurgery, Cleveland, v. 85, p. 154-165, 2016.

PRADA, I. L. S. Neuroanatomia Funcional em Medicina Veterinária com correlações clínicas. Editora Terra Molhada, 2014. 616 p.

PORTUGAL, J. O. et al. Morfometria do encéfalo e formação do circuito arterioso em coelhos da Nova Zelândia. Biotemas, Florianópolis, v. 27, n. 2, p. 147-155, 2014.

RECKZIEGEL, S. H.; LINDEMANN, T.; CAMPOS, R. A. Systematic study of the brain base arteries in capybara (Hydrochoerus hydrochaeris). Brazilian Journal of 
Morphological Science, São Paulo, v.18, n. 2, p. 104-110, 2001.

TESTUT, L. Traité de anatomie humaine. Paris: Octave Doin, 1911. 1052p.

Recebido em: 10.11.2018

Aceito em: 24.03.2019 